\title{
Pseudospectral apparent horizon finders: An efficient new algorithm
}

\author{
Carsten Gundlach * \\ Max-Planck-Institut für Gravitationsphysik, Albert-Einstein-Institut, Schlaatzweg 1, 14473 Potsdam, Germany
}

(Received 23 July 1997; published 19 November 1997)

\begin{abstract}
We review the problem of finding an apparent horizon in Cauchy data $\left(\Sigma, g_{a b}, K_{a b}\right)$ in three space dimensions without symmetries. We describe a family of algorithms which includes the pseudospectral apparent horizon finder of Nakamura et al. and the curvature flow method proposed by Tod as special cases. We suggest that other algorithms in the family may combine the speed of the former with the robustness of the latter. A numerical implementation for Cauchy data given on a grid in Cartesian coordinates is described, and tested on Brill-Lindquist and Kerr initial data. The new algorithm appears faster and more robust than previous ones. [S0556-2821(98)01502-1]
\end{abstract}

PACS number(s): 04.25.Dm

\section{INTRODUCTION}

An important task in numerical relativity is locating black holes in numerically generated spacetimes, both for technical purposes and for extracting physical information. A black hole is a region of spacetime out of which no null geodesics escape to infinity. The boundary of the black hole, the event horizon, is formed by those outward-going, future-directed null geodesics which neither fall into the singularity nor escape to null infinity. The event horizon contains important geometric information about the spacetime. It is a global construction and can in principle only be determined when the entire spacetime is known. In practice, one can obtain a good approximation to the event horizon within a finite spacetime region, once the black hole has settled down to a stationary state. By definition, the event horizon repels future-directed null geodesics, but attracts past ones. One can then evolve past-directed null geodesics back through the spacetime, and find the event horizon as the surface to which they are attracted [1].

Locating black holes is crucial in numerical relativity also for a technical reason: Spacetime slicings which avoid black holes rapidly become singular. Instead one would like to excise a spacetime region just inside the event horizon from the numerical domain during the numerical evolution, using the fact that it cannot influence events outside the black hole. During the time evolution, however, one does not yet know where the event horizon is. Instead one needs to use the poor man's event horizon, the apparent horizon.

An apparent horizon (AH) is defined within a single time slice, or spacelike hypersurface $\Sigma$, namely as a smooth embedded 2-surface whose outgoing normal null geodesics have zero expansion. There may be one such surface enclosing another one, in which case the outermost one is the apparent horizon. If one combines the apparent horizon on each time slice into a 3-dimensional surface, this world tube will depend on the slicing, and can be discontinuous. Nevertheless one can show that if an apparent horizon exists on a given time slice, it must be inside a black hole [2]. The converse is not true: there are slicings of black hole space-

*Electronic address: gundlach@aei-potsdam.mpg.de times without any apparent horizons [3]. For numerical purposes one simply hopes that this case is unusual and that the apparent horizon gives a reasonable indication of the event horizon.

A wide variety of numerical algorithms for finding AHs have been explored or suggested. For the purpose of excising the black hole region, one needs to find the apparent horizon frequently, perhaps at each time step. When two black holes collide, a new AH enveloping the two separate ones appears suddenly. Therefore the main requirements are speed and finding the AH from scratch, without a good initial guess. Precision is less important for black hole excision, although a safe error estimate is, so that one can be sure not to excise too much and inject unphysical boundary conditions.

In spherical symmetry, the $\mathrm{AH}$ problem reduces to an algebraic equation. In axisymmetry, it reduces to an ordinary differential equation with periodic boundary conditions. In this paper we shall be concerned exclusively with the 3dimensional (3D) problem without any symmetries, either continuous or discrete, where one deals with a highly nonlinear elliptic problem on a closed 2-surface. In practice this will always be the 2-sphere, or several disconnected 2spheres [4], which can be treated separately.

All 3D AH finder algorithms proposed so far can be classified according to a few key choices, which can be made independently one from another. How are candidate AHs represented? One can parameterize an embedded 2-surface either by introducing coordinates on it, or as a level set of a function on the 3-dimensional slice in which it is embedded. How is the curvature of the candidate AH calculated? One can discretize the necessary spatial derivatives by finite differencing, finite elements, or pseudospectral methods. A third fundamental choice is between solving the elliptic problem directly, or converting it into a parabolic problem, in which the solution of the elliptic problem is approached during an evolution in an unphysical time parameter. The distinction between these last two approaches is not sharp in practice. On the one hand one always solves a nonlinear elliptic problem by iteration. On the other, numerical implementation of any parabolic approach requires an implicit "time" step for stability, thus posing a new elliptic problem that becomes equivalent to the original one in the limit of an 
infinitely large time step. We now discuss previous AH finders in terms of these choices.

Nakamura, Koshima and Oohara [5] represent the AH in spherical coordinates as $r=h(\theta, \varphi)$. We note that this requires the surface to be a 2-sphere, and star-shaped (convex) around the point $r=0$. The shape function $h(\theta, \varphi)$ is expanded in spherical harmonics. This spectral decomposition is used to calculate the derivatives of $h$ in formulating the elliptic problem. The orthonormality and completeness of the spherical harmonics is used to subtract the linear elliptic operator $L^{2}$ from the nonlinear elliptic problem and invert it. This gives rise to an iteration prescription. We shall see that this iteration can also be described as the discretization in unphysical time of a parabolic problem. It remains unclear from [5] by what method the spectral decomposition back and forth is carried out for Cauchy data which are only known in numerical form and on a grid. The Nakamura et al. algorithm has been independently coded and tested, and extended in various directions by Kemball and Bishop [6]. They report exponential convergence, good robustness, and high precision unless the point $r=0$ is close to the AH.

Tod [7] has proposed a geometrically defined flow under which a trial 2-surface evolves to the $\mathrm{AH}$. For timesymmetric slices, the $\mathrm{AH}$ problem reduces to that of finding a minimal surface, and Tod's prescription to mean curvature flow. This is well-known to converge to minimal surfaces. On non-time-symmetric slices, only lower order terms are added to the problem, so that one may hope that Tod's flow also converges for such data in practice. Tod's algorithm is parabolic, without specifying how the surface is represented or differenced. Tod's algorithm has been implemented numerically by Bernstein [8] using finite differencing in coordinates introduced on the surface. He discusses stable extrinsic algorithms for parabolic problems, and reports good results in axisymmetry using one of them, but technical problems to do with finite differencing on the sphere in the general case. Pasch [9] has implemented mean curvature flow representing the test surface as a level set. This allows for a change of topology during the evolution. He has successfully tested the algorithm using Brill-Lindquist data for 1,2 or 3 black holes, using a fast implicit time evolution package, and finite differencing on a Cartesian grid in the embedding space.

Thornburg [10] attacks the elliptic problem directly using finite differencing on a square $(\theta, \varphi)$ grid, and Newton's method to solve the discretized equations. He calculates the Jacobian required for Newton's method by first linearizing the differential equations, then finite differencing the result. This is more efficient than numerical differentiation. He finds high precision results, but a nonlinear instability in highfrequency modes. Huq [11] has extended Newton's method to data without symmetries in Cartesian-type coordinates.

The NCSA/WashU algorithm $[12,13]$ uses the parameterization $r=h(\theta, \varphi)$, and a spectral decomposition to parameterize $h$ and calculate its derivatives. The discretized elliptic problem is solved by applying a standard minimization algorithm to the sum of $H^{2}$ over all surface points. The spectral basis is not required to be orthonormal for this purpose. Baumgarte et al. [14] have implemented the NCSA/WashU algorithm independently, with the difference that they use the true spherical harmonics as a basis. Both algorithms lo- cate points on the 2-surface on a square $(\theta, \varphi)$ grid, interpolating data from the 3-dimensional Cartesian grid used in the $3+1$ time evolution.

In this paper we review different previous algorithms in one common, fully covariant notation. This analysis suggests to us a new algorithm which combines essential ideas of the algorithms of Tod and Nakamura et al. From our analysis we expect this algorithm to be as fast as that of Nakamura et al. (and therefore much faster than existing implementations of Tod's algorithm), while being as robust in practice as that of Tod. We describe the details of a numerical implementation of this algorithm, and some initial tests. The results are encouraging.

The paper is organized as follows. In Sec. II we set up the mathematical formalism of the problem. We begin by deriving the differential equation that determines an apparent horizon in II A. In II B we discuss different ways of parameterizing apparent horizon candidates, that is, smooth embedded 2-surfaces, and in II C we provide tools for spectral methods on the 2-sphere. In Sec. III we review various algorithms for finding AHs, namely the pseudospectral algorithm of Nakamura et al. [5], Jacobi's method, and the generalized mean curvature flow suggested by Tod [7]. We then build on this review by presenting a family of algorithm which contains the previous algorithms as limiting cases, and suggesting that in the middle of the family there are algorithms that perform better than the limiting members. In Sec. IV we describe a numerical implementation of our proposed algorithm. In Sec. $\mathrm{V}$ we test its performance in finding apparent horizons in Brill-Lindquist and Kerr data given in Cartesian coordinates on a grid.

\section{MATHEMATICAL PRELIMINARIES}

\section{A. The apparent horizon equation}

Here we give a brief derivation (see also [15]) of the differential equation that we try to solve in the remainder of the paper, both to give a complete presentation of the problem and to fix notation. Throughout the paper, lower-case Latin indices from the beginning of the alphabet indicate abstract index notation. Indices from the middle of the alphabet indicate 3-dimensional tensor components. Our signature convention is $(-+++)$.

We begin with a series of definitions. Let $\left(M,{ }^{(4)} g_{a b}\right)$ be a spacetime, and $\nabla_{a}^{(4)}$ the covariant derivative associated with ${ }^{(4)} g_{a b}$. (We use this notation to reserve the symbols $g_{a b}$ and $\nabla_{a}$ for 3 dimensions.) Let $\Sigma$ be a smooth spacelike hypersurface, and let $n^{a}$ be the future-pointing unit timelike normal to $\Sigma$. Then ${ }^{(4)} g_{a b}$ gives rise to Cauchy data

$$
g_{a b}={ }^{(4)} g_{a b}+n_{a} n_{b}, \quad K_{a b}=-g_{a}^{c} \nabla_{c}^{(4)} n_{b}=-\nabla_{a} n_{b},
$$

on $\Sigma$, where $g_{a b}$ is the positive definite 3-metric induced on $\Sigma$ and $K_{a b}$ is the extrinsic curvature of $\Sigma . \nabla_{a}$ is the covariant derivative associated with $g_{a b}$. Let $S$ be a closed smooth hypersurface of $\Sigma$, which means it is two-dimensional and spacelike, and $s^{a}$ its unit outward pointing normal in $\Sigma$, which is also spacelike, and normal to $n^{a} \cdot g_{a b}$ induces a positive definite 2-metric

$$
m_{a b}=g_{a b}-s_{a} s_{b}={ }^{(4)} g_{a b}+n_{a} n_{b}-s_{a} s_{b}
$$


on $S$. Let $k^{a}$ be the future-pointing null geodesic congruence whose projection on $\Sigma$ is orthogonal to $S$, that is

$$
k^{a} \nabla_{a}^{(4)} k^{b}=0, \quad k_{a} k^{a}=0,\left.\quad m_{a b} k^{a}\right|_{S}=0 .
$$

Then $k^{a}$ describes light rays leaving $S$ normally from the point of view of an observer whose instantaneous simultaneity is $\Sigma$. Clearly $k^{a}$ depends not only on the spacetime and on $S$, but also on $\Sigma$. Let $H$ be the expansion of that congruence,

$$
H=\nabla_{a}^{(4)} k^{a} .
$$

We would like to express $H$ in terms of the Cauchy data $\left(\Sigma, g_{a b}, K_{a b}\right)$, and the normal $s^{a}$ to $S$. The crucial step is to note that, up to an overall factor,

$$
\left.k^{a}\right|_{S}=s^{a}+n^{a} .
$$

Clearly this obeys the conditions $m_{a b} k^{a}=0$ and $k_{a} k^{a}=0$ on $S$. We continue $k^{a}$ away from $S$ by the remaining condition $k^{a} \nabla_{a}^{(4)} k^{b}=0$. We also continue $s^{a}$ away from $S$ in $\Sigma$ assuming that it retains unit length, but otherwise in an arbitrary manner. Then we have, on $S$,

$$
\begin{aligned}
H= & { }^{(4)} g^{a b} \nabla_{a}^{(4)} k_{b}=\left(g^{a b}-n^{a} n^{b}\right) \nabla_{a}^{(4)} k_{b} \\
= & g^{a b} \nabla_{a}^{(4)}\left(s_{b}+n_{b}\right)-\left(k^{a}-s^{a}\right)\left(k^{b}-s^{b}\right) \nabla_{a}^{(4)} k_{b} \\
= & g^{a b} \nabla_{a}^{(4)} s_{b}+g^{a b} \nabla_{a}^{(4)} n_{b}-\left(k^{b}-s^{b}\right)\left[k^{a} \nabla_{a}^{(4)} k_{b}\right] \\
& +s^{a}\left[k^{b} \nabla_{a}^{(4)} k_{b}\right]-s^{a}\left[s^{b} \nabla_{a}^{(4)} s_{b}\right]-s^{a} s^{b} \nabla_{a}^{(4)} n_{b} \\
= & \nabla_{a} s^{a}-K+s^{a} s^{b} K_{a b},
\end{aligned}
$$

where $K={ }^{(4)} g^{a b} K_{a b}=g^{a b} K_{a b}$ is the trace of the extrinsic curvature. All terms in square brackets vanish individually by definition.

A smooth embedded closed surface with outward pointing unit normal $s^{a}$ that obeys $H=0$ everywhere on $S$ is called a marginally outer trapped surface. The outermost of such surfaces, if one or more exist in $\Sigma$, is called the apparent horizon in $\Sigma$ [2]. On the one hand this definition is global in $\Sigma$, which makes finding an apparent horizon a nontrivial problem. On the other, it is local in time, as $H$ depends only on the Cauchy data $\left(g_{a b}, K_{a b}\right)$ on a single slice $\Sigma$. If one fixes the slicing of a given spacetime, calculates the apparent horizon on each slice, and then combines the apparent horizons on each slice to obtain a timelike, $2+1$ dimensional worldtube, this world-tube depends on the slicing. This is in contrast to the event horizon, which depends globally on the entire spacetime, but is independent of the slicing.

\section{B. Characterizing closed two-surfaces}

Before we can discuss solving the apparent horizon equation $H=0$ in practice, we need to parameterize candidate apparent horizons, that is, two-dimensional, smooth, closed surfaces $S$ embedded in $\Sigma$.

Let $x^{i}$ be coordinates on $\Sigma$. One way of parameterizing $S$ is then to introduce coordinates $\xi^{A}$ on $S$ (at least locally), and give a map $x^{i}=X^{i}\left(\xi^{A}\right)$. In this case, the topology of $S$ is fixed in advance. Furthermore, different functions $X^{i}$ describe the same abstract surface $S$, corresponding to a change of coordinates $\xi^{A}$ on $S$.
A different way of parameterizing $S$ is as a level set

$$
F\left(x^{i}\right)=0 .
$$

As long as the form of $F\left(x^{i}\right)$ is not restricted, this has the advantage of $S$ allowing to have arbitrary topology. In particular, $S$ can be disconnected. Again, many different functions $F\left(x^{i}\right)$ describe the same abstract surface $S$, as long as they have the one level set $F\left(x^{i}\right)=0$ in common.

It is straightforward to express $H$ as a function of $F$ and its derivatives. The unit normal (with respect to the 3-metric $g_{a b}$ ) of any level set of $F$ is

$$
s^{a}=|\nabla F|^{-1} g^{a b} \nabla_{b} F, \quad \text { where }|\nabla F| \equiv\left(g^{a b} \nabla_{a} F \nabla_{b} F\right)^{1 / 2} \text {. }
$$

Direct substitution now gives

$$
\begin{aligned}
H & =\left(g^{a b}-|\nabla F|^{-2} \nabla^{a} F \nabla^{b} F\right)\left(|\nabla F|^{-1} \nabla_{a} \nabla_{b} F-K_{a b}\right) \\
& =m^{a b}\left(|\nabla F|^{-1} \nabla_{a} \nabla_{b} F-K_{a b}\right) .
\end{aligned}
$$

$H$ is therefore a quasilinear second order differential operator acting on $F$.

Now we come back to the problem that different functions $F(x, y, z)$ describe the same abstract surface $S$. A possible gauge condition would be to make $F$ harmonic with respect to a background metric $\bar{g}_{a b}$, or with respect to the physical 3-metric $g_{a b}$. Then its value everywhere depends only on its value on a suitable two-dimensional surface, such as the boundary of the numerical domain. Here, instead, we follow several previous authors in restricting $F$ to the form

$$
F\left(x^{i}\right)=r\left(x^{i}\right)-h\left[\theta\left(x^{i}\right), \varphi\left(x^{i}\right)\right],
$$

where $(r, \theta, \varphi)$ are related to a set of Cartesian coordinates $x^{i}$ in the usual way, namely $x=r \sin \theta \cos \varphi, y=r \sin \theta \sin \varphi$ and $z=r \cos \theta$. The overall sign of $F$ has been chosen so that $s^{a}$ given in (8) points outward. This parameterization is equivalent to $X^{i}(\theta, \varphi)=x^{i}[r=h(\theta, \varphi), \theta, \varphi]$. The obvious disadvantages of restricting $F$ to this form are that the topology of $S$ must be $S^{2}$, and that $S$ must be star-shaped around the coordinate origin $r=0$. The advantages are that surfaces $S$ correspond uniquely to functions $h$, and that we can use the natural basis $\left\{Y_{l m}\right\}$ for expanding the function $h$.

Considered as a quasilinear differential operator acting on $F\left(x^{i}\right), H$ is not elliptic in three dimensions, because one of the three eigenvalues of $m_{b}^{a}$, the one with eigenvector $s^{a}$, is zero. Considered as a differential operator in two dimensions acting on $h(\theta, \varphi)$, it is elliptic. In this two-dimensional interpretation it is nonlinear not only through the explicit appearance of $\nabla_{a} F$ in the coefficients of $\nabla_{a} \nabla_{b} F$, but also through its dependence on the point where the tensor fields $g_{a b}$ and $K_{a b}$ are evaluated, which depends on $F$ itself. This means that $g_{a b}$ and $K_{a b}$ play the same role in the apparent horizon equation as the internal metric of a nonlinear $\sigma$-model does in its equation of motion. Because the coefficients of the elliptic equation contain $g_{a b}$ and $K_{a b}$ as free functions, it appears unlikely that one can prove existence of solutions for sufficiently general $g_{a b}$ and $K_{a b}$. 


\section{Geometric characterization of the $L^{2}$ operator and spherical harmonics}

In this subsection we introduce in geometric terms some tools that we need later on to discuss spectral methods on the 2 -sphere. The key idea of any pseudospectral method for solving a nonlinear elliptic problem is to subtract from the nonlinear one a simple linear elliptic operator that can be inverted explicitly by spectral methods. In our problem, the principal part of the operator $H$ acting on $h$ is the Laplacian with respect to the 2-dimensional metric $m_{a b}$ induced on the surface $F=0$ by the metric $g_{a b}$. As $F=0$ is topologically a 2 -sphere, a natural candidate for subtraction is the Laplacian $L^{2}$ on the round 2-sphere. It can be inverted using the spherical harmonics.

We could define $L^{2}$ on $\Sigma$ by first introducing spherical coordinates $(r, \theta, \varphi)$, and then defining its action on a scalar $f$ as the usual combination of partial derivatives with respect to those coordinates, that is,

$$
L^{2} f=f_{, \theta \theta}+\cot \theta f_{, \theta}+\sin ^{-2} \theta f_{, \varphi \varphi} .
$$

Setting up these coordinates also has the effect of lifting the spherical harmonics from the 2-sphere to all of $\Sigma$, by smoothly identifying points on different spheres $r=$ const.

The minimal geometric structure which allows us to make the same definitions without reference to preferred coordinates is a flat background metric $\bar{g}_{a b}$ on $\Sigma$ (independent of the physical metric $g_{a b}$ ), together with a preferred point $O$. Let the covariant derivative associated with $\bar{g}_{a b}$ be $\bar{\nabla}_{a}$, and let $\bar{g}^{a b}$ be the inverse of $\bar{g}_{a b}$. We foliate $\Sigma$ into level surfaces of the scalar field $r$, where $r(p)$ is the geodesic distance with respect to $\bar{g}_{a b}$ between the points $p$ and $O$. The vector $r^{a} \equiv \bar{g}^{a b} \bar{\nabla}_{b} r$ is the unit normal with respect to $\bar{g}_{a b}$ on the surfaces of constant $r$. The flat metric $g_{a b}$ then induces the metric $\bar{g}_{a b}-\bar{\nabla}_{a} r \bar{\nabla}_{b} r$ on the surfaces of constant $r$. This induced metric has a constant curvature of $r^{-2}$, so that $r^{-2}\left(\bar{g}_{a b}-\bar{\nabla}_{a} r \bar{\nabla}_{b} r\right)$ is a metric of unit curvature on the 2spheres $r=$ const. We now define $L^{2}$ as the Laplacian of this 2-dimensional metric:

$$
L^{2}=r^{2}\left(\bar{g}^{a b}-r^{a} r^{b}\right) \bar{\nabla}_{a} \bar{\nabla}_{b}-2 r r^{a} \bar{\nabla}_{a}
$$

By direct substitution one verifies that, if $\bar{g}_{a b}$ is given as

$$
d s^{2}=d r^{2}+r^{2}\left(d \theta^{2}+\sin ^{2} \theta d \varphi^{2}\right),
$$

this reduces to Eq. (11). Our definition (12), however, is covariant, and can be used to define the action of $L^{2}$ on arbitrary tensors, and in arbitrary coordinate systems.

For our purposes we characterize the spherical harmonics $Y_{l m}$ as a set of scalar functions on $\Sigma$ with two properties: They are orthonormal in the sense that

$$
\int_{S} Y_{l m}^{*} Y_{l^{\prime} m^{\prime}} d \Omega=\delta_{l l^{\prime}} \delta_{m m^{\prime}}
$$

where $S$ is any smooth surface that is star-shaped around $r$ $=0$, and where $d \Omega$ is the measure induced on $S$ by $r^{-2}\left(\bar{g}_{a b}-\bar{\nabla}_{a} r \bar{\nabla}_{b} r\right)$. (In spherical coordinates this reduces to the standard measure $d \Omega=\sin \theta d \theta d \varphi$.) From this it follows that

$$
r^{a} \bar{\nabla}_{a} Y_{l m}=0
$$

[In spherical coordinates $Y_{l m}=Y_{l m}(\theta, \varphi)$.] We also require that the $Y_{l m}$ are eigenfunctions of $L^{2}$ :

$$
L^{2} Y_{l m}=-l(l+1) Y_{l m}
$$

for $l=0,1,2, \ldots$ and $m=-l, \ldots, l$. We do not define $m$ as the eigenvalue of $L_{z}$ ( $\partial / \partial \varphi$ in spherical coordinates), but only use it as a label on the orthonormal basis. This leaves us free to combine $Y_{l m}$ and $Y_{l,-m}$ of the standard complex definition to obtain a real orthonormal basis more convenient for numerical purposes.

\section{ALGORITHMS FOR SOLVING THE APPARENT HORIZON EQUATION}

\section{A. The Nakamura et al. algorithm}

We now use our covariant notation for $L^{2}$ and the $Y_{l m}$ in reviewing the algorithm of Nakamura, Kojima and Oohara [5] (NKO) for finding an apparent horizon. NKO characterize $S$ by $r=h(\theta, \varphi)$ in spherical coordinates, and expand $h$ in spherical harmonics:

$$
h(\theta, \varphi)=\sum_{l=0}^{l_{\max }} \sum_{m=-l}^{l} a_{l m} Y_{l m}(\theta, \varphi) .
$$

(A finite value of $l_{\max }$ is required in any numerical implementation.) We begin our description of the algorithm with the trivial observation that $H=0$ is equivalent to

$$
\rho H+L^{2} h=L^{2} h
$$

where $\rho$ is any strictly positive function. In the NKO algorithm, the weight function $\rho$ is specified by demanding that the coefficient of the partial derivative $h_{, \theta \theta}$ cancels in the combination $\rho H+L^{2} h$. (The notation $\rho$ is ours, not that of NKO. We introduce it here because we want to consider other choices of $\rho$ later on.) Integrating over the $Y_{l m}$ and using (14) and (16), we obtain

$$
\int_{S} Y_{l m}^{*}\left(\rho H+L^{2} h\right) d \Omega=-l(l+1) a_{l m} .
$$

NKO now use this equation in an iteration procedure, $\left\{a_{l m}\right\}^{(n)} \rightarrow\left\{a_{l m}\right\}^{(n+1)}$, where $(n)$ labels iteration steps, of the form

$$
a_{l m}^{(n+1)}=-\frac{1}{l(l+1)} \int_{S} Y_{l m}^{*}\left(\rho H+L^{2} h\right)^{(n)} d \Omega,
$$

where the right-hand side is evaluated from the $\left\{a_{l m}\right\}^{(n)}$. As this formula does not cover $a_{00}, a_{00}$ is determined at each iteration step by solving

$$
\int_{S}\left(\rho H+L^{2} h\right) d \Omega=0
$$


for $a_{00}$ (note $Y_{00}^{*}=$ const).

We now try to understand what makes the NKO method work. For this purpose we express $H(h)$ in terms of the flat background derivation $\bar{\nabla}_{a}$ :

$$
\begin{aligned}
H= & \left(g^{a b}-s^{a} s^{b}\right)\left\{|\nabla F|-1\left[\bar{\nabla}_{a} \bar{\nabla}_{b} F-\frac{1}{2} g^{c d}\left(\bar{\nabla}_{a} g_{c b}+\bar{\nabla}_{b} g_{a c}\right.\right.\right. \\
& \left.\left.\left.-\bar{\nabla}_{c} g_{a b}\right) \bar{\nabla}_{d} F\right]-K_{a b}\right\}
\end{aligned}
$$

where $F \equiv r-h(\theta, \varphi)$, and $s^{a}$ is defined by Eq. (8). Because $r^{a} \bar{\nabla}_{a} h=0$ by definition, we have

$$
L^{2} h=r^{2}\left(\bar{g}^{a b}-r^{a} r^{b}\right) \bar{\nabla}_{a} \bar{\nabla}_{b} h .
$$

Putting Eqs. (22) and (23) together, and keeping in mind that $F=r-h$, we obtain

$$
\rho H+L^{2} h=M^{a b} \bar{\nabla}_{a} \bar{\nabla}_{b} h+W,
$$

where $M^{a b}$ and $W$ depend explicitly on first derivatives of $h$, and implicitly on $h$ through the point in $\Sigma$ where $g_{a b}$ and $K_{a b}$ are evaluated. We have quietly assumed that $\rho$ does not depend on second or higher derivatives of $h$, so that $\rho H$ $+L^{2} h$, like $H$ itself, acts on $h$ as a quasilinear second-order differential operator. This is indeed the case for the $\rho$ of NKO and the other choices we explore later on. The principal symbol $M^{a b}$ is

$$
M^{a b}=-\rho|\nabla F|^{-1}\left(g^{a b}-s^{a} s^{b}\right)+r^{2}\left(\bar{g}^{a b}-r^{a} r^{b}\right) .
$$

The principal symbol of a quasilinear differential operator does not depend on the choice of derivation, here $\bar{\nabla}_{a}$. We can verify this for the case at hand.

We see that $M^{a b}$ is a difference between two projectors: the first one onto surfaces of constant $F$, and with respect to the physical metric $g_{a b}$, the other onto surfaces of constant coordinate $r$, and with the respect to the background metric $\bar{g}_{a b}$. In the trivial case where $g_{a b}$ is conformally related to $\bar{g}_{a b}$ (conformally flat), and where surfaces of constant $F$ coincide with surfaces of constant $r$ (spherical symmetry), one can choose $\rho$ so as to make the entire tensor $M^{a b}$ vanish. In general, one can impose only one condition on its six components. The choice of NKO is, in our notation,

$$
M^{\theta \theta}=0 .
$$

We prefer a coordinate-independent choice, and impose

$$
M^{a b}\left(\bar{g}_{a b}-\bar{\nabla}_{a} r \bar{\nabla}_{b} r\right)=0 .
$$

The motivation of either choice is to cancel, as far as possible, that part of $M^{a b} \bar{\nabla}_{a} \bar{\nabla}_{b}$ which looks like $L^{2}$. Our choice does not introduce a preferred direction within the tangent space of $S$, which may be an esthetic more than a practical advantage. Solving our condition for $\rho$, we obtain

$$
\rho=2 r^{2}|\nabla F|\left[\left(g^{a b}-s^{a} s^{b}\right)\left(\bar{g}_{a b}-\bar{\nabla}_{a} r \bar{\nabla}_{b} r\right)\right]^{-1} \equiv|\nabla F| \sigma,
$$

where the second equation defines $\sigma$.
Now we recognize an important ingredient of the NKO algorithm, its smoothing property. Putting the individual components $a_{l m}$ back together again, we can write (20) as

$$
h^{(n+1)}=\left(L^{2}\right)^{-1}\left(\rho H+L^{2}\right) h^{(n)} .
$$

(This is only formal because of the special role of $a_{00}: L^{2}$ does not have an inverse.) Any iterative algorithm for solving an elliptic problem runs the danger of being unstable to the growth of high-frequency numerical noise. Whereas $H$ acts on $h$ as a second-order differential operator, thus increasing unsmoothness, $\rho H+L^{2}$ has the $L^{2}$ part taken out, and therefore creates less high-frequency noise. Moreover, $\left(L^{2}\right)^{-1}$ acts as a smoothing operator. One therefore expects $h^{(n+1)}$ to be smoother than $h^{(n)}$. This is a necessary property for any iterative algorithm that can converge from a rough initial guess without blowing up through high-frequency noise on the way.

\section{B. Jacobi's method, and stability}

In order to see how the NKO algorithm is related to other algorithms, we rewrite (29) once more, as

$$
h^{(n+1)}-h^{(n)}=\left(L^{2}\right)^{-1}(\rho H)^{(n)} .
$$

It is now tempting to go from the discrete algorithm to a continuous flow in an unphsical "time" parameter $\lambda$ :

$$
\frac{\partial h(\theta, \varphi, \lambda)}{\partial \lambda}=\left(L^{2}\right)^{-1} \rho H(h) .
$$

The NKO algorithm proper, namely

$$
a_{l m}^{(n+1)}-a_{l m}^{(n)}=-\frac{1}{l(l+1)}(\rho H)_{l m}^{(n)}, \quad l>0,
$$

is formally recovered from this differential equation by forward-differencing it with respect to $\lambda$, with a step size $\Delta \lambda=1$, and inverting $L^{2}$ by the pseudospectral method (we again disregard the special role of $a_{00}$ ). Other differencing methods, such as centered differencing, and using a different "time" step, give rise to obvious alternative algorithms. Some of these have been examined by Kemball and Bishop [6]. Kemball and Bishop also consider different methods of enforcing the constraint (21) on $a_{00}$ and of coupling it to the iteration method for the other $a_{l m}$.

Any flow method can be considered as an example of Jacobi's method. This is the recipe of solving an elliptic equation $E(h)=0$ by transforming it into a parabolic equation $\partial h / \partial \lambda=E(h)$. If $E$ is the Laplace operator, then the resulting equation is the heat equation, and Jacobi's method is known to converge. As $H$ acting on $h$ resembles $-L^{2}$, one might try the flow

$$
\frac{\partial h}{\partial \lambda}=-H(h)
$$

We have implemented this numerically in the pseudospectral framework and find empirically that its high frequency noise blows up unless one chooses a very small step size.

The origin of this instability is clear from the analogy with the heat equation. The heat equation on $S^{2}$ is 


$$
f_{, \lambda}=L^{2} f
$$

We decompose $f$ into spherical harmonics, as $f(\theta, \varphi, \lambda)$ $=\Sigma f_{l m}(\lambda) Y_{l m}(\theta, \varphi)$. For the spectral components we obtain $d f_{l m} / d \lambda=-l(l+1) f_{l m}$. All spectral components decrease exponentially. Discretizing this equation in time, however, for example by forward differencing, we obtain $f_{l m}^{(n+1)}$ $=f_{l m}^{(n)}-\Delta \lambda l(l+1) f_{l m}^{(n)}$. This is stable only for $\mid 1-\Delta \lambda l(l$ $+1) \mid<1$, that is for $\Delta \lambda<2 / l(l+1)$. In all explicit methods, the time step is limited by order of magnitude to

$$
\Delta \lambda \lesssim \frac{1}{l_{\max }\left(l_{\max }+1\right)} .
$$

The same limit arises if one discretizes $L^{2}$ by finite differencing, where it takes the form $\Delta \lambda \lesssim(\Delta \theta)^{2} \simeq(\Delta \varphi)^{2}$. Similar stability limits exist for all parabolic equations. The NKO algorithm does not have this instability problem. It replaces $H=0$ by $\left(L^{2}\right)^{-1}(\rho H)=0$ as the elliptic problem to be solved, and clearly $\left(L^{2}\right)^{-1}$ acts a smoothing operator that keeps high frequency noise down. An appropriate choice of $\rho$ makes this even more effective by making $\rho H$ as similar to $-L^{2}$ as possible.

\section{Mean curvature flow}

From considering an iterative approach as the discretization of a flow on the space of surfaces, one is led to the generalized mean curvature flow algorithm of Tod [7] and other geometrically motivated flows. Tod proposes deforming a trial surface $S$ embedded in $\Sigma$ by means of the flow

$$
\left(\frac{\partial}{\partial \lambda}\right)^{a}=-s^{a} H,
$$

where $s^{a}$ is again the outward-pointing normal to $S$. [Tod uses the notation $d x^{i} / d \lambda$ for the left-hand side, but we wanted to stress here that $(\partial / \partial \lambda)^{a}$ is a vector field and independent of coordinates.] For time-symmetric Cauchy data, $K_{a b}=0$, we have $H=\nabla_{a} s^{a}$, which is simply the trace of the extrinsic curvature of $S$ induced by its embedding in $\Sigma$, also called the mean curvature. $H=0$ is then equivalent to $S$ having extremal area, and $s^{a} H$ is the gradient of the area. In this case, mean curvature flow is guaranteed to converge to a surface of $H=0$, or extremal area, also called a minimal surface. There is an extended literature on mean curvature flow and minimal surfaces [16]. Tod's idea is to generalize this method from $H=\nabla_{a} s^{a}$ to $H=\nabla_{a} s^{a}-K+K_{a b} s^{a} s^{b}$. For $K_{a b} \neq 0$, this flow is no longer guaranteed to converge, but one may hope that it does, as the additional terms are of lower order.

One essential strength of generalized mean curvature flow is that it cannot move a test surface through an $\mathrm{AH}$, even for $K_{a b} \neq 0$. The argument is simple [17]: Assume that the test surface is about to move through the true $\mathrm{AH}$, that is, it touches it at one point. At that point both surfaces see the same $g_{a b}, K_{a b}$ and $s^{a}$. Of the quantities which go into the expression (6) for $H$, only the $\nabla_{a} s^{a}$ differ on the two surfaces. Keeping track of the signs, one sees that the test surface must then always back away from the true $\mathrm{AH}$ at that point. Therefore, a smooth test surface can never cross an
$\mathrm{AH}$ (although it can approach it asymptotically). This is true not only for generalized mean curvature flow, but for all flows of the form $(\partial / \partial \lambda)^{a}=-s^{a} \rho H$, as long as $\rho$ is strictly positive. This property allows us to start the algorithm on a large surface far out and evolve it inwards, thus making sure we find the true $\mathrm{AH}$.

We note that Eq. (36) does not only specify the deformation of $S$ as an abstract surface, but also identifies any point on $S$ with a point on its deformation. That information is not essential to the method, and we get rid of it if we define $S$ as the level set $F=0$. Then $s^{a}$ is again given by (8). Consider a family of moving surfaces $S(\lambda)$ given by $F\left(x^{i}, \lambda\right)=0$. On the surface $F=$ const we must have

$$
\frac{d F}{d \lambda} \equiv \frac{\partial F}{\partial \lambda}+\left(\frac{\partial}{\partial \lambda}\right)^{a} \nabla_{a} F=0
$$

and therefore

$$
\frac{\partial F}{\partial \lambda}=-\left(\frac{\partial}{\partial \lambda}\right)^{a} \nabla_{a} F=s^{a} H \nabla_{a} F=|\nabla F| H .
$$

We note that (36) is a geometric prescription: It specifies a vector field on $S$ only in terms of the geometry of $S$ and $\Sigma$ and the tensor field $K_{a b}$, independently of how $S$ is parameterized. As we have just shown, the parabolic equation (38) is equivalent to it. We conclude that a flow parameterized by, for example, $\partial F / \partial \lambda=H$, without the factor $|\nabla F|$, does not have such a geometric interpretation, but must depend on $F$ in a more general way than only through the shape of its level set $S$. On the other hand, as $H$ is a scalar function of $g_{a b}, s^{a}$ and $K_{a b}$ (evaluated on $S$ ), we can replace it by any other scalar and still obtain a flow with geometric meaning. Any flow of the form

$$
\frac{\partial F}{\partial \lambda}=|\nabla F| \times \text { any } \operatorname{scalar}\left(K_{a b}, g_{a b}, s^{a}\right)
$$

is therefore geometric in nature. Such a general equation, replacing $H$ by any function of the curvature of $S$, has already been given by Osher and Sethian [18].

If we now restrict $F$ to the form $F\left(x^{i}, \lambda\right)=r-h(\theta, \varphi, \lambda)$, we have $\partial F / \partial \lambda=-\partial h / \partial \lambda$. Therefore, any flow of the form

$$
\frac{\partial h}{\partial \lambda}=-|\nabla(r-h)| \times \text { any } \operatorname{scalar}\left(K_{a b}, g_{a b}, s^{a}\right)
$$

is again geometric in nature. The naive Jacobi method, Eq. (33), however, is not.

\section{D. "Fast flow" methods}

Before we propose our own AH finding algorithm, we summarize the strengths and weaknesses of the existing ones. We have not discussed algorithms which attack the elliptic problem directly via Newton's method or a minimization iteration. Their main drawback, however, is a small range of convergence, that is, they require a very good initial guess. NKO is a lot more robust, but the need to treat $a_{00}$ separately is an important disadvantage. Equation (21) is by no means trivial: Solving it by any iterative method like Newton's method is as computationally expensive as many 


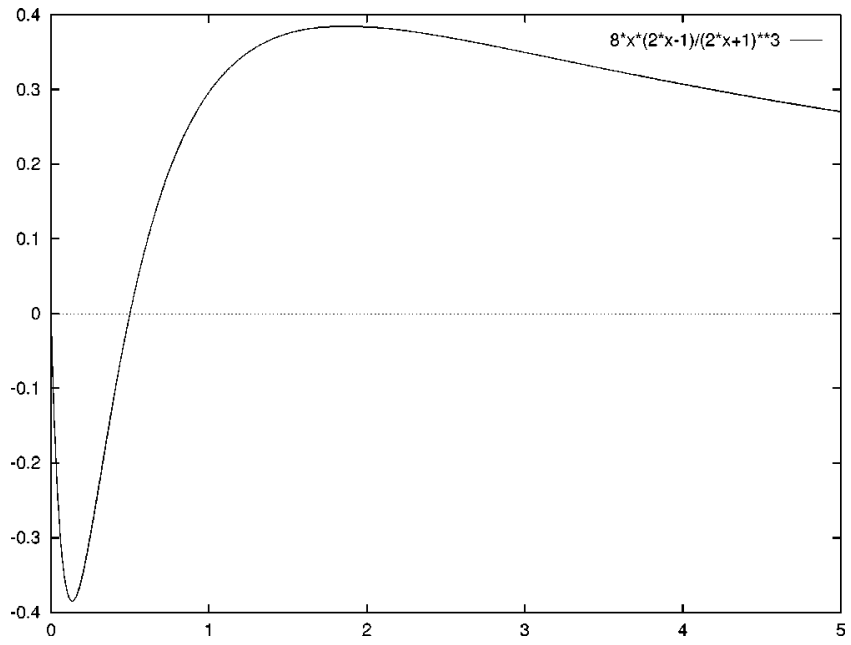

FIG. 1. Plot of the horizon function $H(\bar{r})$ versus $\bar{r}$, in units of the black hole mass $m$, as given in Eq. (41).

steps of the main iteration loop. Furthermore, for given data $\left(g_{a b}, K_{a b}\right)$ and given $a_{l m}, l>0$, there may be several roots $a_{00}$ of this equation, or none. Kemball and Bishop [6] propose investigating each root of the equation separately, or if there are none, each minimum of the right-hand side. Clearly this makes the algorithm even more expensive.

Most importantly, Newton's method for solving (21) tends to go off into the wrong direction. As an example for this problem [19], we consider time-symmetric Cauchy data for the Kruskal spacetime of mass $m$ in isotropic coordinates. [This is the special case $m_{2}=0$ of Eq. (61) below.] As a trial surface we take a sphere of coordinate radius $\bar{r}$ centered on the black hole. The expansion of outgoing null rays is

$$
H=H(\bar{r})=\frac{8 \bar{r}(2 \bar{r}-m)}{(2 \bar{r}+m)^{3}} .
$$

From a mathematical point of view, this example is degenerate in the sense that $H=0$ is a reduced from a differential to a purely algebraic equation by the spherical symmetry of the trial surface. (There is only one spatial direction, and this is the degenerate direction of the elliptic operator.) Nevertheless, any problems that arise in this toy equation also arise in a more realistic situation. From a plot of $H(\bar{r})$, Fig. 1, we see that for $\bar{r} \gtrsim 1.87 m$ Newton's method wanders off to infinity, and for $r \leqq 0.13 m$ goes towards $\bar{r}=0$, instead of finding the zero at $\bar{r}=m / 2$. All algorithms which use Newton's method, or a minimization method using derivatives, for any or all of the $a_{l m}$, that is, the direct elliptic algorithms, share this problem.

The curvature flow method is sensitive only to the sign of $H$, not its derivative. Applied to this problem, it goes towards smaller $r$ for positive $H$, and towards larger $r$ for negative $H$, and always finds the apparent horizon. We have already seen that it cannot accidentally walk through the AH. In these two properties lies its robustness. Any flow with $\rho H$ instead of $H$ on the right-hand side, where $\rho$ is strictly positive and a scalar, shares the fundamental advantages of the generalized mean curvature method: a trial surface far outside the apparent horizon always moves in, and can never accidentally cross the apparent horizon. As we have seen, however, flow methods are slow because explicit discretizations in time of parabolic methods require very small time steps for stability. An implicit time step may be possible, but introduces a new elliptic problem which a priori is not simpler than the underlying elliptic problem one wants to solve.

We are not bound to geometrically motivated flows, however. Instead, we heuristically consider all flow methods as variants of the Jacobi method for solving $H=0$. Then we are free to combine the best features of the curvature flow and NKO methods. From curvature flow we would like to keep the properties that $a_{00}$ is not treated specially, and that the change of $a_{00}$ should be proportional to $H_{00}$, not $d H_{00} / d a_{00}$. From NKO we would like to adopt the idea of subtracting and then inverting $L^{2}$, in order to suppress highfrequency noise. This leads us to the following family of flows:

$$
\frac{\partial h}{\partial \lambda}=-A\left(1-B L^{2}\right)^{-1} \rho H
$$

where $A$ and $B$ are free positive constants, and where $\rho$ is a strictly positive weight depending on $h$ through at most first derivatives. The differential operator $1-B L^{2}$ is invertible, with positive eigenvalues, for $B \geqslant 0$, and for $B>0$ its inverse is a smoothing operator. When we discretize in $\lambda$, we can absorb $\Delta \lambda$ into $A$. For simplicity we also restrict ourselves to forward differencing. In spectral components, we obtain

$$
a_{l m}^{(n+1)}-a_{l m}^{(n)}=-\frac{A}{1+B l(l+1)}(\rho H)_{l m}^{(n)} .
$$

For $\rho$ we consider three choices: $\rho=1$ ("H flow"), $\rho=|\nabla F|$ ("C flow"), and $\rho=|\nabla F| \sigma$ with $\sigma$ defined in (28) ("N flow"). With $A>0$ and $B=0, \mathrm{H}$ flow is the Jacobi method, and $\mathrm{C}$ flow is the curvature flow method. $\mathrm{N}$ flow formally becomes the NKO method [compare Eq. (32)] in the limit $A=B \rightarrow \infty$. The limit is singular because the NKO method is not a flow and has to update the component $a_{00}$ separately.

For determining the optimal values of $A$ and $B$, it is convenient to reparameterize them with new parameters $\alpha$ and $\beta$ as

$$
A=\frac{\alpha}{l_{\max }\left(l_{\max }+1\right)}+\beta, \quad B=\frac{\beta}{\alpha} .
$$

$A$ and $B$ now scale with $l_{\max }$ in such a way that we expect the optimal values of $\alpha$ and $\beta$ to be independent of the value of $l_{\text {max }} \cdot \alpha$ parameterizes an $l$-independent contribution to the effective step size of $\alpha /\left[l_{\max }\left(l_{\max }+1\right)\right]$, while $\beta$ adds an $l$-dependent speedup which is zero at $l=l_{\max }$ and increases to $\beta$ at $l=0$.

It is clear that the fast flow methods have the potential to be much faster than curvature flow, while still being numerically stable and robust against bad initial guesses. They are not really flows of the form (40) because they are not local. In some situations, the effective weight $\rho$ can become negative on parts of the surface, and in these situations, the "fast flow" can move through the true AH. Fast flow methods should be considered as (good) compromises between the robustness of curvature flow and the speed of NKO. Further- 
more, one can trade robustness for speed by increasing $\beta$, and vice versa, and so adapt the algorithm to the situation. We have obtained the best results using $\mathrm{N}$ flow, with $\alpha=1.0$ and $\beta=0.5$ in (44). We note that $\alpha=1.0$, for any $\beta$, means that the algorithm treats high frequency components like the NKO algorithm does.

\section{NUMERICAL IMPLEMENTATION OF PSEUDOSPECTRAL APPARENT HORIZON FINDERS}

The algorithm we suggest in this paper is formally defined by Eq. (43) with $\rho$ defined by (28) and $A \simeq B \simeq 0.5$.

In order to implement this or any other pseudospectral algorithm, we need to calculate the spectral components $(\rho H)_{l m}$ from the spectral coefficients $a_{l m}$. In this section we give details of an algorithm for doing this, given $g^{i j}, K_{i j}$ and $g_{i j, k}$ on a Cartesian grid. We expect that there is scope for increasing the speed and reducing the discretization error in this low-level part of the algorithm, without changing the top-level part given by (43).

\section{A. The background structure}

The parameterization of the surface $S$ through spherical harmonics and the introduction of the differential operator $L^{2}$ require the introduction of a flat metric $\bar{g}_{a b}$. We do this by introducing auxiliary Cartesian coordinates $\bar{x}^{i}=f^{i}\left(x^{j}\right)$, and then setting the components of $\bar{g}_{a b}$ in the coordinates $\bar{x}^{i}$ to be $\delta_{i j}$. The corresponding metric derivation $\bar{\nabla}_{a}$ is then $\partial / \partial \bar{x}^{i}$, and $r^{2}=\delta_{i j} \bar{x}^{i} \bar{x}^{j}$. In these coordinates $L^{2}$ is given by the expression

$$
L^{2}=\left(r^{2} \delta^{i j}-\bar{x}^{i} \bar{x}^{j}\right) \frac{\partial}{\partial \overline{x^{i}}} \frac{\partial}{\partial \bar{x}^{j}}-2 \bar{x}^{i} \frac{\partial}{\partial \bar{x}^{i}} .
$$

While more complicated choices are possible, we define

$$
\overline{x^{i}} \equiv x^{i}-x_{0}^{i}
$$

where $x^{j}$ are the Cartesian coordinates in which the Cauchy data are presented to our algorithm. The freedom to shift the origin $r=0$ around is necessary because any trial surface will have to be star-shaped around $r=0$, that is around $x^{i}=x_{0}^{i}$. Therefore we have to make sure that $x^{i}=x_{0}^{i}$ is inside the $\mathrm{AH}$.

\section{B. Calculating the $Y_{l m}$}

We need to calculate the $Y_{l m}\left(\bar{x}^{i}\right)$ and their first two partial derivatives for arbitrary $\left(\bar{x}^{i}\right)$. Speed is important, because our algorithm spends most of its time in these calculations. The standard spherical harmonics are

$$
Y_{l}^{m}=\bar{P}_{l}^{m}(\cos \theta) e^{i m \varphi}
$$

where the $\bar{P}_{l}^{m}$ are associated Legendre functions times a constant depending on $l$ and $m$. Instead of the complex $Y_{l m}$, we introduce the real basis $\bar{Y}_{l m}$ as

$$
\begin{gathered}
\bar{Y}_{l}^{0}=\bar{P}_{l}^{0}(\cos \theta), \quad \bar{Y}_{l}^{|m|}=\sqrt{2} \bar{P}_{l}^{|m|}(\cos \theta) \cos m \varphi, \\
\bar{Y}_{l}^{-|m|}=\sqrt{2} \bar{P}_{l}^{|m|}(\cos \theta) \sin m \varphi .
\end{gathered}
$$

The real $\bar{Y}_{l m}$ obey the same conditions (14)-(16) as the standard complex $Y_{l m}$, but they are not eigenfunctions of $L_{z}$ $=\partial / \partial \varphi$. At each point $\bar{x}=(x, y, z)$ we calculate

$$
\cos \theta=z / r, \quad \sin \theta=\rho / r, \quad \cos \varphi=x / \rho, \quad \sin \varphi=y / \rho,
$$

where

$$
r=\sqrt{x^{2}+y^{2}+z^{2}}, \quad \rho=\sqrt{x^{2}+y^{2}} .
$$

No explicit evaluation of trigonometric functions is required. Then $\cos m \varphi$ and $\sin m \varphi$ are calculated as polynomials in $\cos \varphi$ and $\sin \varphi$ from the recursion relations

$$
\begin{aligned}
& \cos m \varphi=\cos (m-1) \varphi \cos \varphi-\sin (m-1) \varphi \sin \varphi \\
& \sin m \varphi=\cos (m-1) \varphi \sin \varphi+\sin (m-1) \varphi \cos \varphi
\end{aligned}
$$

The $\bar{P}_{l m}$ are given explicitly for $m=l$ by

$$
\bar{P}_{l}^{l}(\cos \theta)=\frac{1}{\sqrt{4 \pi}} \sqrt{\frac{(2 l+1)(2 l) !}{2^{l} l !}}(-\sin \theta)^{l},
$$

and for $0 \leqslant m \leqslant l-1$ are calculated from the recursion relations

$$
\begin{aligned}
\bar{P}_{l}^{m}(\cos \theta)= & \sqrt{\frac{2 l+1}{l^{2}-m^{2}}}\left[\sqrt{2 l-1} \cos \theta \bar{P}_{l-1}^{m}(\cos \theta)\right. \\
& \left.-\sqrt{\frac{(l-1)^{2}-m^{2}}{2 l-3}} \bar{P}_{l-2}^{m}(\cos \theta)\right] .
\end{aligned}
$$

(They are not needed for $m<0$.) In order to calculate the first and second partial derivatives with respect to $x, y$ and $z$, we calculate the partial derivatives of $\bar{Y}_{l m}$ with respect to $\theta$ and $\varphi$, and those of $\theta$ and $\varphi$ with respect to $x, y$ and $z$, and explicitly code all terms arising from the chain rule. The derivatives of $\bar{P}_{l m}(\cos \theta)$ with respect to $\theta$ are obtained recursively after differentiating Eqs. (52) and (53). The relations (15) and (16) are then obeyed to machine precision by the numerically calculated quantities.

We are aware of two other algorithms for calculating $Y_{l m}\left(\bar{x}^{i}\right)$ and their first and second partial derivatives. The algorithm of Baumgarte et al. calculates them recursively as polynomials of the $r_{, i}$. We have coded this algorithm directly from the detailed formulae in [14], and find that it scales in time as $l_{\max }^{4}$ and in storage requirement as $l_{\max }^{3}$. The NCSA/WashU apparent horizon finder $[12,13]$ does not calculate the $Y_{l m}$, but a related basis of smooth functions. This basis is not orthogonal, and it is not independent of $r$ at constant $\theta$ and $\varphi$. For the NCSA/WashU algorithm these properties of the basis functions are not necessary. The calculation of this basis scales as approximately $l_{\max }^{4}$ in time, and as $l_{\text {max }}^{4}$ in storage [20]. In common with both algorithms, ours is recursive, and does not require trigonometric function 
TABLE I. Maximal deviation of the overlap matrix $A_{n n^{\prime}}$ from the unit matrix as a function of the linear grid size, the integration surface $S$ and $l_{\max }$.

\begin{tabular}{lcccc}
\hline \hline Surface parameters & grid size & $l_{\max }=4$ & $l_{\max }=8$ & $l_{\max }=16$ \\
\hline$a_{00}=1.0$ & 16 & 0.053 & 0.159 & 0.284 \\
& 32 & 0.016 & 0.037 & 0.069 \\
$a_{00}=1.0, a_{1,-1}=0.4$ & 64 & 0.011 & 0.026 & 0.040 \\
$a_{00}=1.0, a_{10}=0.4$ & 32 & 0.039 & 0.069 & 0.178 \\
& 64 & 0.047 & 0.047 & 0.053 \\
& 32 & 0.052 & 0.087 & 0.274 \\
\hline \hline
\end{tabular}

evaluations. The difference is that it breaks up the $Y_{l m}$ into the product of a function of $\theta$ times a function of $\varphi$. In consequence it scales $l_{\max }^{2}$ in time (it is faster already for $l_{\max }=2$ ), and as $l_{\max }^{2}$ in storage requirement. This optimal scaling comes at a price: the algorithm breaks down on the axis $x=y=0$, where cancellations between the $\theta$ and $\varphi$ dependent factors in the analytic expressions fail to take place numerically. In practice, one can evade the problem by moving any collocation points that come very close to the $z$ axis a small distance away from it, resulting in a small error at that point, and a negligible one in the integrals over $S$. Incorporating the cancellations into the code properly requires mixing the $\theta$ and $\varphi$ dependency by going through an intermediate, over-complete basis called "symmetric trace-free tensors," which is precisely the approach of Baumgarte et al.

\section{Interpolating and integrating over $S$}

We need to discretize the integral $\int_{S} d \Omega$. We take as collocation points on $S$ all those points where $S$ intersects a link of the three-dimensional Cartesian grid. A link is the straight line between two neighboring points on the Cartesian grid. The links which intersect $S$ are those on which $F$ changes sign. The advantage of this choice of collocation points is that one only needs to interpolate in one dimension. Furthermore the number of collocation points on $S$ scales with the number of nearby points on the Cartesian grid, that is, with the available numerical information.

To find the surface $F=0$ the algorithm calculates $F$ on all Cartesian grid points. For this it needs the $\bar{Y}_{l m}$ on all grid points. Although these are required again and again, present technology does not allow us to store $l_{\max }\left(l_{\max }+1\right)$ 3D arrays for reasonable $l_{\max }$, so that they have to be recomputed each time. Then the algorithm flags all links on which $F$ changes sign. Both operations scale as $N^{3}$, where $N$ is the linear grid size. We determine by inverse linear interpolation where on the link the intersection point is, then interpolate $g^{i j}, g_{i j, k}$ and $K_{i j}$ to the intersection point by cubic interpolation. We calculate $F_{, i}$ and $F_{, i j}$ directly at the $\overline{x^{i}}$ of the intersection point. For this purpose we need the $r_{, i}$ and the $\bar{Y}_{l m, i}$ and $\bar{Y}_{l m, j}$.

The integral $\int_{S} d \Omega$ is now approximated by the sum

$$
\int_{S} f d \Omega \simeq 4 \pi \frac{\sum w f}{\sum w}
$$

where the sum is over all collocation points. Let $\bar{s}^{i}$ be the unit normal on $S$ with respect to the flat background metric $\bar{g}_{a b}$ :

$$
\bar{s}=\left[\delta^{k l}\left(r_{, k}-h_{, k}\right)\left(r_{, l}-h_{, l}\right)\right]^{-1 / 2} \delta^{i j}\left(r_{, j}-h_{, j}\right) .
$$

The integration weight $w$ is then given by

$$
\begin{aligned}
w= & \frac{d \Omega}{d N}=\frac{d \Omega}{d A} \frac{d A}{d N}, \quad \frac{d \Omega}{d A}=\frac{r_{, i} \bar{s}^{i}}{r^{2}}, \quad \frac{d N}{d A}=\frac{\left|\bar{s}^{1}\right|}{\Delta \overline{x^{2} \Delta} \bar{x}^{3}} \\
& +\frac{\left|\bar{s}^{2}\right|}{\Delta \bar{x} \Delta \bar{x}^{3}}+\frac{\left|\bar{s}^{3}\right|}{\Delta \bar{x} \Delta \bar{x}^{2}},
\end{aligned}
$$

where $\Delta \bar{x}^{i}$ are the grid spacings on the three axes. Here $d \Omega$ is the solid angle with respect to the flat metric $\bar{g}_{a b}$ around $r=0$, that is $d \Omega=\sin \theta d \theta d \varphi, d A$ is the surface element on $S$ induced by $\bar{g}_{a b}$, and $d N$ is the number of intersections of $d A$ with grid links. Note that the expression for $d N / d A$ models the anisotropy of the Cartesian grid in an explicit sum over the three grid directions. The sum (54) is a good approximation to the integral when $\bar{s} i$ changes little from one collocation point to its neighbors.

As a test of the discrete approximation to integrals over the surface, we calculate the overlap integrals (14) numerically. Let us combine the indices $l$ and $m$ of the spherical harmonics into one index $n$. The numerical approximation to the symmetric matrix $A_{n n^{\prime}}=\int Y_{n} Y_{n}, d \Omega$ is not exactly equal to the unit matrix, because of the finite number of collocation points.

One could arrange the weights $w$ such that $A_{n n^{\prime}}$ comes out right for a given set of collocation points, but that would require putting the collocation points in fixed, special positions with respect to $\theta$ and $\varphi$, for example on a square grid in $\theta$ and $\varphi$. In our algorithm, however, we let the position of the collocation points be dictated by the underlying Cartesian grid $\bar{x}^{i}$, and rely on a number of collocation points much larger than the number $n_{\max }=l_{\max }\left(l_{\max }+1\right)$ of basis functions in order to keep the error down. Table I shows how the error in $A_{n n}$, increases with $l$ for a surface $S$ created under realistic conditions by the apparent horizon finder. In practice, the size of the 3-dimensional grids is limited by the available computer storage, so that we have to choose $l_{\max }$ small enough for the spectral error to remain small. 
We can reduce this error in the following way. Let us denote by $H_{n}=H_{n}\left(a_{n^{\prime}}\right)$ the true spectral components of the function $H$ on the surface parameterized by the expansion coefficients $a_{n}$. Let $\widetilde{H}_{n}$ be their numerical, slightly incorrect value. Clearly we have

$$
\widetilde{H}_{n}=\sum_{n^{\prime}=1}^{\infty} A_{n n^{\prime}} H_{n^{\prime}}
$$

Without much additional numerical work, we can calculate a finite square piece of the infinite matrix $A$ when we calculate $\widetilde{H}_{n}$ up to $n_{\max }$. Let $B$ be the inverse of that finite part of $A$. Then we have (for $n \leqslant n_{\max }$ )

$$
\begin{aligned}
\hat{H}_{n} & \equiv \sum_{n^{\prime}=1}^{n_{\max }} B_{n n^{\prime}} \widetilde{H}_{n^{\prime}}=H_{n}+\sum_{n^{\prime}=1}^{n_{\max }} B_{n n^{\prime}}\left(\sum_{n^{\prime \prime}=n_{\max }+1}^{\infty} A_{n^{\prime} n^{\prime \prime}} H_{n^{\prime \prime}}\right) \\
& \simeq H_{n}+\sum_{n^{\prime}=n_{\max }+1}^{\infty} A_{n n^{\prime}} H_{n^{\prime}} .
\end{aligned}
$$

In $\hat{H}_{n}$ the unwanted aliasing among the low $\left(n \leqslant n_{\max }\right)$ frequencies has been eliminated, and the remaining deviation from the true value $H_{n}$ comes only from the aliasing of high frequencies to low ones. One would assume this to be a better approximation to $H_{n}$ than the $\widetilde{H}_{n}$ in normal situations. In practice, however, this assumption is difficult to test, as no cheap estimate of the error $\hat{H}_{n}-H_{n}$ is available.

Still, there are some indications of the remaining error in the $\hat{H}_{n}$ : The spectral components of $r$, the $r_{n}$, are by definition identical to $a_{n}$. We find that the $\hat{r}_{n}$ are much closer to $a_{n}$ than the $\widetilde{r}_{n}$, but do not converge to them. The remaining error can only be due to the fact that the collocation points do not lie exactly on the true surface $S$ parameterized by the $a_{n}$, due to the interpolation used to find them. This failure to find the true surface $S$ is the only source of error for the $\hat{r}_{n}$, but appears to be also the dominant source of error for the $\hat{H}_{n}$, or any other nontrivial function on $S$. In practice we proceed as follows: We use $\hat{H}_{n}$ as our best approximation to $H_{n}$. We monitor convergence of the final result $a_{n}$ of the AH finder with $l_{\max }$ and the grid spacing of the underlying Cartesian grid. We also monitor $\left|\hat{r}_{n}-a_{n}\right|$. Finally, and perhaps most importantly, we find that the algorithm using $\bar{H}_{n}$ converges better, and its error is considerably reduced when tested against data for which the apparent horizon is known in closed form. Therefore we always use the $\hat{H}_{n}$ and other hatted quantities in the algorithm.

In order to estimate the quantity

$$
\int_{S} H^{2} d \Omega=\sum_{n=1}^{\infty} H_{n}^{2}
$$

which indicates to what precision the algorithm has found the apparent horizon, we use the two numerically available quantities

$$
\left(H_{\mathrm{rms}}\right)^{2} \equiv \frac{\Sigma H^{2} w}{\sum w}=\sum_{n=1}^{\infty} \sum_{m=1}^{\infty} A_{n m} H_{n} H_{m}
$$

and

$$
|H|^{2} \equiv \sum_{n=1}^{n_{\max }} \hat{H}_{n}^{2}
$$

After this work was carried out, we became aware of a different, perhaps more efficient algorithm for going back between a function of $\theta$ and $\phi$ and its spherical harmonic components [21]. One puts a grid on $S$ which is rectangular and equally spaced in $\theta$ and $\varphi$, and then uses fast Fourier transforms in $\theta$ and $\phi$. In a second step, one has to discard those linear Fourier components which are not sufficiently regular at the poles $\theta=0, \pi$, which is rather complicated. In order to evaluate $g_{i j}$ etc. at the collocation points required now, one has to interpolate in three dimensions from the given Cartesian grid, instead of in one dimension. Nevertheless, there may be scope for a more efficient algorithm here.

\section{TESTS}

\section{A. Brill-Lindquist data}

The NCSA/WashU algorithm appears to be the only one to have been tested on numerically evolved data [13]. Tests that use data given in closed form avoid interpolation from numerical data on a grid, which poses an additional source of numerical error and even instability in realistic situations. In the present paper we test our algorithm with data given in closed form, but passed to the algorithm only on the points of a numerical grid of realistic size. The input into the code are the numerical values of the inverse metric and extrinsic curvature components and of the first partial derivatives of the metric components on the grid. A performance test with data derived both from numerical initial data algorithms and numerical time-evolutions is left to a future publication.

As a first test of the complete AH finder, we use BrillLindquist time-symmetric initial data. For two black holes, these are

$$
K_{i j}=0, \quad g_{i j}=\left(1+\frac{m_{1}}{2\left|x-x_{1}\right|}+\frac{m_{2}}{2\left|x-x_{2}\right|}\right)^{4} \delta_{i j} .
$$

The generalization to $N$ black holes is clear.

We begin with a single black hole, where the $\mathrm{AH}$ is known explicitly: it is a coordinate sphere of radius $m / 2$. There are a number of possible convergence criteria for the iterative algorithm, none of which fits all possible situations. One such criterium is $H_{\text {rms }}>2|H|$. [These measures were defined in (60).] This means that the residual of $H(\theta, \varphi)$ is mainly in the high frequencies that we do not resolve. Table II shows the performance of the algorithm for this convergence criterium. We chose a grid spacing such that there are roughly 16 grid points across the interior of the $\mathrm{AH}$. By the standards of a $3 \mathrm{D}$ single grid numerical relativity code on a current supercomputer that is already as much resolution as one can hope for. We chose $l_{\max }=6$, which is roughly the optimal value for that resolution. The initial data are always $a_{00}=0.8$, while the horizon radius is 0.5 . (For convenience, 
TABLE II. Root-mean-square residuals of $H$ and maximal errors in the position of the numerically calculated AH, for Schwarzschild data offset from the coordinate origin. The coordinate radius of the $\mathrm{AH}$ is 0.5 .

\begin{tabular}{lccc}
\hline \hline Offset & iterations & $H_{r m s}$ & $(\Delta r)_{\max }$ \\
\hline 0.0 & 10 & $9 \times 10^{-4}$ & $7 \times 10^{-4}$ \\
0.1 & 10 & $9 \times 10^{-4}$ & $7 \times 10^{-4}$ \\
0.2 & 11 & $1 \times 10^{-3}$ & $8 \times 10^{-4}$ \\
0.3 & 12 & $2 \times 10^{-3}$ & $1 \times 10^{-3}$ \\
0.4 & 10 & $6 \times 10^{-2}$ & $2 \times 10^{-2}$ \\
\hline \hline
\end{tabular}

we use $a_{l m}$ rescaled by a factor of $\sqrt{4 \pi}$, so that $a_{00}$ is the average coordinate radius of the $\mathrm{AH}$.)

We have varied the offset of the center of the spherical harmonics from the center of the AH. $\Delta r$ is the error in locating the $\mathrm{AH}$ in coordinate space. It is calculated directly in those points where the $\mathrm{AH}$ is collocated by the algorithm, not from the $a_{l m}$. The result is roughly independent of the direction of the offset. We see that if the surface is very eccentric around the origin of coordinates, precision suffers. Fortunately, there is a simple remedy: If the dipole moments $a_{1}^{0, \pm 1}$ are large, the algorithm automatically uses them to obtain a better value for the origin $x_{0}^{i}$ of coordinates, and restarts. This source of error is totally eliminated by the procedure. The same remedy applies if the surface touches the origin of coordinates at any stage during the flow.

The next test is Brill-Lindquist data for two uncharged black holes of equal mass. We can position the two centers $x_{1}$ and $x_{2}$ so that the metric is symmetric with respect to the $x=0, y=0$ and $z=0$ planes. This allows us to work numerically on an octant of the full grid, and save time and storage. The situation is in fact axisymmetric, but the code does not
TABLE III. Root-mean-square residuals of $H$ on the inner and outer numerically calculated minimal surface in Brill-Lindquist data for two black holes of equal mass. "No convergence", is the unaided return status of the algorithm. It means that the residual value of $H$ given in brackets is not due to a lack of numerical resolution.

\begin{tabular}{lcc}
\hline \hline Separation & inner $H_{\text {rms }}$ & outer $H_{\text {rms }}$ \\
\hline 0.0 & only one surface & $1.9 \times 10^{-5}$ \\
0.4 & $8 \times 10^{-2}$ & $1.8 \times 10^{-5}$ \\
0.8 & $9 \times 10^{-5}$ & $1.8 \times 10^{-5}$ \\
1.2 & $3.0 \times 10^{-4}$ & $1.5 \times 10^{-4}$ \\
1.4 & $2.6 \times 10^{-4}$ & $2.0 \times 10^{-3}$ \\
1.6 & $2.8 \times 10^{-4}$ & no convergence $\left(3.0 \times 10^{-2}\right)$ \\
1.8 & $2.4 \times 10^{-4}$ & no convergence $\left(3.9 \times 10^{-1}\right)$ \\
2.0 & $7 \times 10^{-4}$ & (not attempted) \\
\hline \hline
\end{tabular}

know that. In Fig. 2 all points on the discretely represented surface are plotted, giving coordinates $z$ versus $\rho$ $=\sqrt{x^{2}+y^{2}}$. The fact that they all fall on one curve shows that the code represents an axisymmetric surface well in spite of the underlying Cartesian grid.

In the data (61) one can always find two minimal surfaces surrounding $x_{1}$ and $x_{2}$. If $x_{1}$ and $x_{2}$ are close enough together, there is a third minimal surface surrounding both of them. Determining the maximal separation at which this happens is not an easy test. Assume that the two centers are just far enough apart that there no longer is a common horizon. By continuity there will still be a smooth surface on which $H$ is small, but not zero, everywhere. Numerically, this cannot be distinguished from a true horizon.

In the test, the two black holes have equal mass parameters $m_{1}=m_{2}=1$. The total ADM mass is 2 . We look for both inner and outer surfaces. In Table III we show, for the same numerical parameters, the root-mean-squared value of

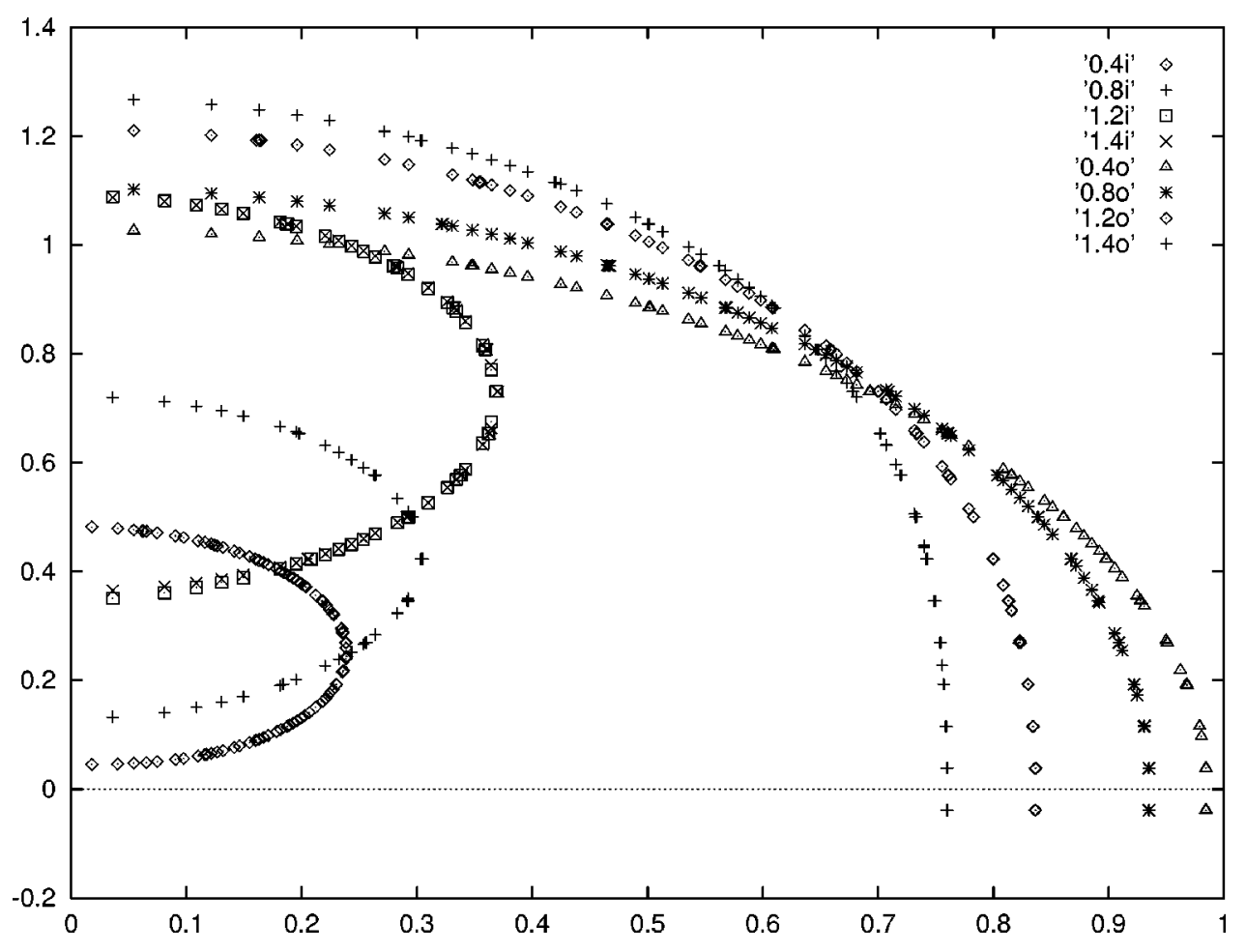

FIG. 2. Shape of the AH in the axisymmetric, z-reflectionsymmetric situation. The algorithm assumes $\mathrm{x}, \mathrm{y}$ and $\mathrm{z}-$ reflection symmetry, but not axisymmetry. The plot shows $z$ versus $\sqrt{x^{2}+y^{2}}$ for all grid points on the $\mathrm{AH}$ in one octant of the full grid. The small half circles are the inner horizons, for a separation of $d=0.4,0.8,1.2$ and 1.4 of the two black holes, from bottom to top. The large quarter circles are the outer horizons, from right to left, or bottom to top. 
$H$ on the trial surface after our algorithm has stopped, against the (coordinate) separation $d$ of the two centers $x_{1}$ and $x_{2}$. The axisymmetric numerical algorithm of Brill and Lindquist does not find an outer minimal surface for $d>1.56$. From our calculations we can say with confidence that the limit lies between 1.4 and 1.6. We should stress again that this precision is limited by the resolution of the Cartesian grid on which we give the Cauchy data. An algorithm specialized to axisymmetry could of course determine this limit with much higher precision.

\section{B. Kerr data in Cartesian coordinates}

In order to test our code on analytic data with nonvanishing extrinsic curvature, we consider Kerr data. Cauchy data for the Kerr spacetime have been given by Brandt and Seidel [22]. We transform these to Cartesian coordinates by defining $x^{i} x^{k} \delta_{i j}=\bar{r}^{2}$, where $\bar{r}$ is the radial coordinate which generalizes the isotropic radial coordinate $\bar{r}$ for the Schwarzschild spacetime. For testing our algorithm it is useful if we do not restrict the angular momentum vector, or symmetry axis, to the $z$-axis, but give its direction as a unit vector $n^{i}$, $n^{i} n^{j} \delta_{i j}=1$. The transformed expressions are

$$
\begin{gathered}
g_{i j}=A\left(\delta_{i j}+B v_{i} v_{j}\right), \quad g^{i j}=A^{-1}\left(\delta^{i j}-\frac{B}{1+v^{k} v_{k} B} v^{i} v^{j}\right), \\
K_{i j}=v_{i} w_{j}+w_{i} v_{j}, \\
v^{i}=\epsilon^{i j k} n_{j} x_{k}, \quad w^{i}=C x^{i}+D\left(n^{i}-\cos \theta x^{i}\right)
\end{gathered}
$$

where $\cos \theta=n^{k} x_{k} / \bar{r}$, where all indices are moved with $\delta_{i j}$, and where the coefficients are

$$
\begin{gathered}
A=\rho^{2} \bar{r}^{-2}, \quad B=\left(\rho^{2}+2 m r\right) a^{2} \rho^{-2} \bar{r}^{-4} \\
C=\left[\left(r^{2}+a^{2}\right)^{2}-\Delta a^{2} \sin ^{2} \theta\right]^{-1 / 2} a m\left[2 r^{2}\left(r^{2}+a^{2}\right)+\rho^{2}\left(r^{2}\right.\right. \\
\left.\left.-a^{2}\right)\right] \rho^{-3} \bar{r}^{-4}, \\
D=\left[\left(r^{2}+a^{2}\right)^{2}-\Delta a^{2} \sin ^{2} \theta\right]^{-1 / 2} 2 a^{3} m r \Delta^{1 / 2} \cos \theta \rho^{-3} \bar{r}^{-4} \\
\rho^{2}=r^{2}+a^{2} \cos ^{2} \theta, \quad \Delta=r^{2}-2 m r+a^{2} \\
r=m+\bar{r}+\frac{m^{2}-a^{2}}{4 \bar{r}} .
\end{gathered}
$$

The apparent horizon is the coordinate sphere $\bar{r}$ $=\sqrt{m^{2}-a^{2}} / 2$. For $a=0$ these data reduce to Brill-Lindquist data for a single black hole. While $K_{a b}$ does not vanish, the data are still special in that the two contributions to $H, \nabla_{a} s^{a}$ and $m^{a b} K_{a b}$, vanish separately on the AH. We have tested our algorithm on these data for different ratios of $a / m$, different offsets $x_{0}^{i}$ between the center of the black hole and the center of spherical harmonics, and for different orientations $n^{i}$ of the black hole symmetry axis relative to the spherical harmonics. The results are essentially the same as for the single Brill-Lindquist black hole, giving an indication that the presence of the extrinsic curvature term does not make a qualitative change to the performance of the algorithm.

\section{CONCLUSIONS}

Numerical general relativity requires a fast and robust algorithm for finding apparent horizons in Cauchy data without symmetries in three dimensions given on a grid. In this paper we have described a new apparent horizon finder algorithm which appears to be as fast but more robust than its best predecessor.

We began from a general classification of possible approaches to the problem. Any approach which poses the problem as a nonlinear elliptic equation on a topological two-sphere and then attacks that equation directly will fail unless provided with a very good initial guess, because the problem is nonlocal in nature. While we have disregarded such approaches here, they will be ideal as a second stage whenever the apparent horizon needs to be determined to high precision. We concluded that for robustness the best algorithm is probably the (generalized) mean curvature flow suggested by Tod [7], where an arbitrary initial surface evolves in an unphysical "time", towards the apparent horizon, turning the problem from an elliptic into a parabolic one. The algorithm is guaranteed to converge at least for time-symmetric $\left(K_{a b}=0\right)$ data, and we have argued that it must be at least very robust also for $K_{a b} \neq 0$ data. Unfortunately, numerical implementations of this algorithm face a numerical stability problem common to all parabolic equations, which make them slow, and increasingly so with increasing resolution, in practice.

This stability or speed problem is not present in the algorithm of Nakamura et al. (NKO) [5]. It is motivated by a standard way of solving nonlinear elliptic problems numerically, namely subtracting a simple linear elliptic operator from the nonlinear one, inverting it by pseudo-spectral methods and iterating. Here we have thrown more light on how NKO works, by making explicit the background metric it introduces, and by characterizing the iteration procedure as a specific finite differencing, in unphysical time, of a parabolic problem. This parabolic problem itself is the singular limit of a certain family of flows which are governed by a mixture of the physical geometry of the Cauchy data and an unphysical background geometry. Tod's flow is a different limiting case of that family, one in which no background metric appears.

Once we have recognized the existence of a continuum of possible algorithms between Tod and NKO, it is plausible that an algorithm somewhere in the middle of the continuum may be better than the extremes. By trial and error, we have determined the optimal member of the family of algorithms. This intermediate algorithm evolves the high-frequency components (the fine details) of the trial AH essentially like the NKO algorithm, but it evolves the low frequency components (the rough shape) by a variant of generalized mean curvature flow. We therefore call it "fast flow.'

We have given details of a numerical implementation of the pseudo-spectral methods which are needed for implementing both the original NKO and our new algorithm. Such details have not been published before. It should be stressed that the formal analysis of the algorithm in Sec. III is independent from its implementation in Sec. IV, and there may be different and more efficient implementations.

We have not made direct performance comparisons with other algorithms, and the tests we have described are viabil- 
ity rather than performance tests. Nevertheless, we anticipate the following:

By construction, the new algorithm is as fast as that of NKO: The iteration steps are very similar, and there is the same small number of them. NKO, however, updates $a_{00}$ (the overall radius of the trial $\mathrm{AH}$ ) by a special procedure. According to how this is done [6], the additional overhead may be large. More importantly, the separate update of $a_{00}$ has the potential to reduce the robustness of NKO: Eq. (21) may have several solutions, in which case all should be investigated, or none, in which case minima should be investigated instead [6]. This requires some decision-taking, which will be hard to automate, or instead an infinitely branching search. We have also argued that zeros of Eq. (21) are hard to find. Either NKO or fast flow should be far more tolerant of initial guesses than the elliptic methods.

The method of choice for robustness and elegance is clearly Tod's mean curvature flow. The only question here is speed. We have argued that as a parabolic method this would be slow in the possible implementations known to us, but a quantitative comparison with the implementation of Pasch [9] would be interesting.
The only existing algorithm directly accessible to the author is that of the NCSA/WashU group $[12,13]$. Direct comparisons are planned in future realistic applications. The new algorithm seems to be more robust, though: it made the transition from two AHs to a single one in the family of BrillLindquist data summarized in Table I without external input. This ability will be crucial for applying AH boundary conditions in the merger of two black holes when the computer code is on its own during a very large run. The NCSA/ WashU code has not been tested on a similar sequence of analytic initial data, but in some situations involving evolved black holes it presently requires some care in finding the correct horizon [23].

Finally, the source code of the new spectral AH finder will be published early in 1998 in conjunction with the "Cactus" numerical relativity infrastructure [24].

\section{ACKNOWLEDGMENTS}

I am grateful to Bernd Bruegmann and Steve Brandt for helpful discussions on all aspects of this paper, and to Ed Seidel for a critical reading.
[1] P. Anninos et al., Phys. Rev. Lett. 74, 630 (1995); J. Libson et al., Phys. Rev. D 53, 4335 (1996).

[2] R. M. Wald, General Relativity (University of Chicago Press, Chicago, 1984).

[3] R. M. Wald and V. Iyer, Phys. Rev. D 44, R3719 (1991).

[4] G. W. Gibbons, Commun. Math. Phys. 27, 87 (1972).

[5] T. Nakamura, Y. Kojima, and K. Oohara, Phys. Lett. 106A, 235 (1984).

[6] A. J. Kemball and N. T. Bishop, Class. Quantum Grav. 8, 1361 (1991).

[7] K. P. Tod, Class. Quantum Grav. 8, L115 (1991).

[8] D. Bernstein, "Notes on the mean curvature flow method for finding apparent horizons" (unpublished).

[9] E. Pasch, "The level set method for the mean curvature flow on $\left(\mathbf{R}^{3}, g\right)$ ', (unpublished).

[10] J. Thornburg, Phys. Rev. D 54, 4899 (1996).

[11] M. Huq, A finite difference method for locating apparent horizons in 3D Cartesian coordinates, talk at the Numerical Relativity Workshop at Pennsylvania State University, 1993 (unpublished).

[12] J. Libson et al., in The Seventh Marcel Grossmann Meeting on Recent Developments in Theoretical and Experimental General Relativity, Gravitation, and Relativistic Field Theories,
Proceedings, Stanford, California, 1994, edited by R. Jantzen et al. (World Scientific, Singapore, 1966), gr-qc/9412058.

[13] P. Anninos et al., "Finding Apparent Horizons in Dynamic 3D Numerical Spacetimes," gr-qc/9609059.

[14] T. W. Baumgarte et al., Phys. Rev. D 54, 4849 (1996).

[15] J. York, in Frontiers in Numerical Relativity, edited by C. Evans, L. Finn, and D. Hobill (Cambridge University Press, Cambridge, England, 1989).

[16] G. Huisken, Proc. Symp. Pure Math. 54 (1993), Part I.

[17] R. Bartnik (personal communication).

[18] S. Osher and J. A. Sethian, J. Comput. Phys. 79, 12 (1988).

[19] S. Brandt (personal communication).

[20] I thank Peter Anninos for making his FORTRAN code for the calculation of "symmetric trace-free tensors" available. The scaling with $l_{\max }$ can be inferred already from the papers $[12,13]$.

[21] A. H. Norton, in Proceedings of the First Australasian Conference on General Relativity and Gravitation, edited by D. L. Wiltshire (University of Adelaide, Adelaide, 1996), p. 39-47.

[22] S. R. Brandt and E. Seidel, Phys. Rev. D 54, 1403 (1996).

[23] K. Camarda and E. Seidel (personal communication).

[24] J. Massó and P. Walker, in preparation. See also the WWW page cactus.aei-potsdam.mpg.de. 